\section{A mutation in the iron-responsive element of ALAS2 is a modifier of disease severity in a patient suffering from CLPX associated erythro- poietic protoporphyria}

Porphyrias are a group of eight genetically distinct disorders, each resulting from a partial deficiency or gain-offunction of a specific enzyme in the heme biosynthetic pathway. ${ }^{1}$ Porphyrias are inherited as autosomal dominant, autosomal recessive or X-linked traits. ${ }^{2}$ Erythropoietic protoporphyria (EPP) is a constitutive hematological disorder characterized by protoporphyrin IX (PPIX) accumulation in erythrocytes and other tissues resulting in acute skin photosensitivity, mild microcytic anemia, and rarely, severe liver disease. The majority of the patients with EPP present the autosomal EPP form (OMIM \#177000) due to a partial deficiency of ferrochelatase $(\mathrm{FECH})$, the last enzyme of the heme biosynthetic pathway. ${ }^{1,2}$ In most EPP patients, the clinical expression requires the coinheritance of a $F E C H$ mutation, that abolishes or markedly reduces $\mathrm{FECH}$ activity, in trans to an hypomorphic FECH allele (rs2272783, NM_000140.3; c.[315-48T>C]) carried by about $11 \%$ of Caucasians. $^{3}$ In Europe and the USA, $4-10 \%$ of EPP patients have been reported to harbor a gain-of-function mutation in the $11^{\text {th }}$ exon of the erythroid $\delta$-aminolevulinic synthase gene (ALAS2). ${ }^{4}$ Rare cases of EPP have also been reported in few reference centers without any mutations in the FECH or ALAS2 genes (personal communication from JC Deybach). EPP due to gain-of-function ALAS2 mutations are inherited as an X-linked trait and result in a distinct biochemical feature not only with overproduction and accumulation of free PPIX but also zinc protoporphyrin ( $\mathrm{ZnPP}$ ) (XLPP) (OMIM \#300751). In these patients, the FECH enzyme is functional and utilizes all available iron for heme production. The excess PPIX is used to make ZnPP in a reaction catalyzed by FECH. ${ }^{3,5}$ Moreover, gain-of-function missense mutations altering the C-terminal part of ALAS2 exacerbate congenital erythropoietic porphyria, suggesting that ALAS2 is a gatekeeper of erythroid heme biosynthesis and may function as a modifier gene.
The 5' untranslated region (UTR) of ALAS2 mRNA contains a cis-regulatory iron-responsive element (IRE) that confers iron-dependent posttranscriptional regulation by the iron regulatory proteins (IRP). ${ }^{7}$ IRE mutations are known to cause human diseases. IRE mutations in ferritin L mRNA cause hereditary hyperferritinemia with cataract syndrome (HHCS) (OMIM \#600886), ${ }^{8-9}$ and a single point mutation in the IRE of ferritin $\mathrm{H}$ is responsible for an autosomal dominant iron overload phenotype (OMIM \#615517). ${ }^{10}$ These cases suggest a possible role for IRE mutations in the ALAS2 gene in modifying the severity of hematologic diseases.

Here, we describe an 18 year-old Caucasian female proband (III:2, Figure 1A) referred to the French Center of Porphyria because of early onset (9 months) acute photosensitivity characterized by painfully phototoxic reactions suggesting EPP. An incomplete genetic characterization of this case was previously reported to harbor a gain of function in the mitochondrial unfoldase gene, CLPX. ${ }^{11}$ Written informed consent was obtained for all participants. This study was approved through the local ethical committees in accordance with the World Medical Association Declaration of Helsinki ethical principles for medical research involving human subjects and its subsequent amendments (R162-16-7 and 145-15-4 French ethical agreement). In the proband, a high level of free erythrocyte PPIX and ZnPP confirmed the diagnosis of EPP (Table 1). At the age of diagnosis, the proband also presented with a microcytic iron deficiency anaemia (Table 1). FECH enzyme activity was normal (Table 1). No point mutation or large FECH gene deletion were identified, and chromosome 18, where $F E C H$ gene is located, was excluded by linkage and comparative genomic hybridization (CGH) array analyses. Moreover, the proband did not harbor the FECH low-expressed allele rs2272783, NM_000140.3; c.[31548T >C] (IVS3-48C). The father (II.4) and one uncle (II.2) of the proband presented with zinc- and free PP accumulation in erythrocytes that were associated with a mild photosensitivity, but without symptoms of EPP (Figure 1 and Table 1). Whole-exome sequencing (WES) analysis showed that proband (III.2), the father (II.4) and the uncle (II.2) carried a heterozygous single

Table 1. Clinical and biochemical data in affected subjects of the erythropoietic protoporphyria proband's family.

\begin{tabular}{|c|c|c|c|c|c|c|c|c|}
\hline Pedigree & II:2 & II:4 & \multicolumn{2}{|c|}{ ||l|:2 } & $\pi: 5$ & 1.3 & II:6 & Normal values \\
\hline Sex & M & M & \multicolumn{2}{|c|}{$\mathrm{F}$} & $\mathrm{F}$ & $\mathrm{F}$ & $\mathrm{F}$ & \\
\hline Photosensitivity & mild & mild & \multicolumn{2}{|c|}{ Severe } & no & no & no & \\
\hline IRE ALAS2 mutation & - & - & \multicolumn{2}{|c|}{+} & + & + & + & \\
\hline Age at the visit (years) & 56 & 46 & 18 & 20 & 43 & 78 & 51 & \\
\hline Total porphyrins in plasma (nmol/L) & 54 & 52 & 936 & 780 & 22 & 19 & 20 & $<20$ \\
\hline Erythroid PPIX ( $\mu \mathrm{mol} / \mathrm{L}$ RBC): & 30.4 & 26.7 & 140.9 & 89.5 & 2.1 & 1.9 & 2.0 & $<1.9$ \\
\hline Free PPIX (\%) & 33 & 38 & 71 & 65 & 20 & 17 & 22 & $<28$ \\
\hline $\mathrm{ZnPP}(\%)$ & 67 & 62 & 29 & 35 & 80 & 83 & 78 & $>72$ \\
\hline FECH activity (nmol/mg prot. h) & 4.4 & 3.9 & 3.6 & 3.9 & 4.3 & 4.2 & 4.2 & $>3.5$ \\
\hline $\mathrm{Hb}(\mathrm{g} / \mathrm{dL})$ & 15.5 & 15.9 & 10.9 & 12.1 & 12.2 & 13.3 & 14.0 & $11.5-16.0$ \\
\hline Serum iron $(\mu \mathrm{mol} / \mathrm{L})$ & 25.0 & 20.0 & 4.0 & 11.0 & 21.0 & 14.0 & 20.0 & $12.0-26.0$ \\
\hline Serum ferritin $(\mu \mathrm{g} / \mathrm{L})$ & 196 & 171 & 5 & 11 & 12 & 137 & 85 & $\begin{array}{l}15-250 \text { 오 } \\
8-150 \text { 우 }\end{array}$ \\
\hline Transferrin saturation (\%) & 31 & 32 & 5 & 15 & 31 & 24 & 36 & $20-45$ \\
\hline Soluble Transferrin receptor (mg/L) & 1.24 & 1.41 & 3.95 & 1.75 & 1.15 & 1.26 & 1.19 & $0.76-1.76$ \\
\hline
\end{tabular}

The proband (III:2) was examined twice: first at the initial visit associated with iron deficiency and one year later after oral iron supplementation.PPIX: protoporphyrin IX ZnPP: Zinc protoporphyrin; Hb: hemoglobin; FECH: ferrochelatase; RBC: red blood cells; m: male; f: female. 
A

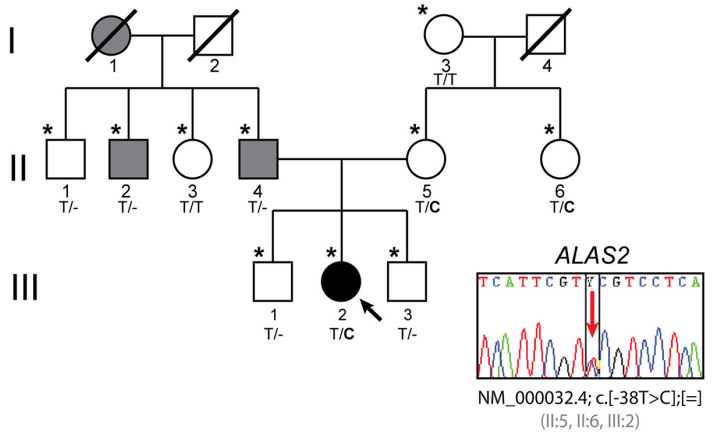

C
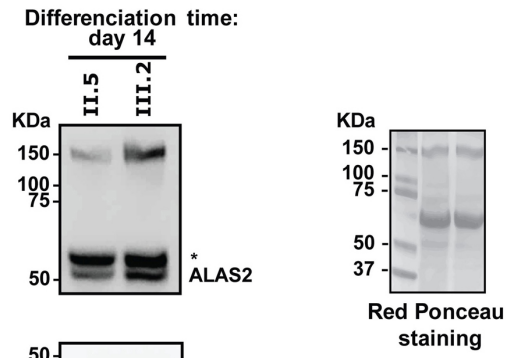

B
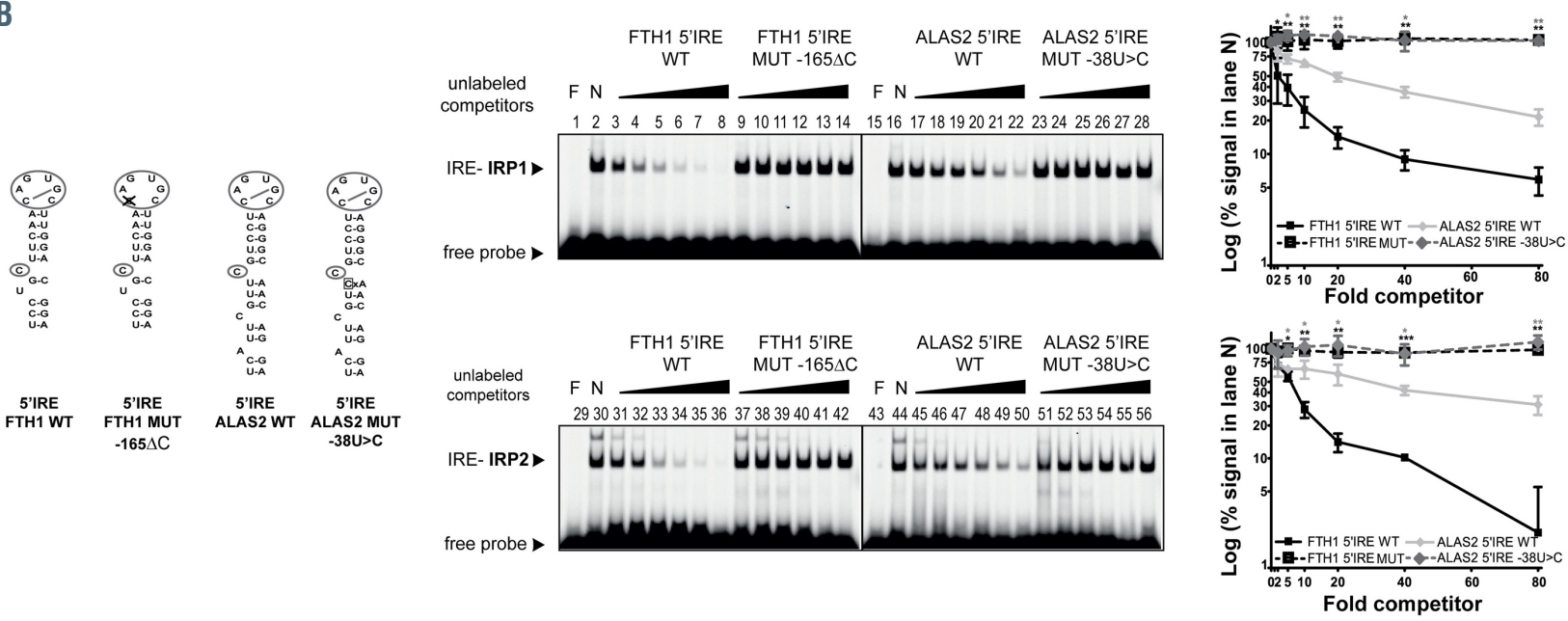

Figure 1. ALAS2 iron-responsive element mutation as modifier for erythropoietic protoporphyria clinical severity. (A) Pedigree of the studied family. Filled black symbol indicates the patient with clinical overt erythropoietic protoporphyria (EPP). Grey symbols indicate relatives (II.2 and II.4) with protoporphyrin IX (PPIX) over-production in red blood cells (RBC) associated with mild photosensitivity without EPP; the grandmother (I.1) presented with a suspected mild photosensitivity but no biochemical data were available; barred symbols indicate deceased subjects. Asterisks indicate subjects characterized at the molecular level. Partial chromatograms of ALAS2 iron-responsive element (IRE) sequences where the -38T>C mutation is located. Reference sequence for ALAS2: NM_000032.4. (B) Non-radioactive competitive electrophoretic mobility shift assays (EMSA) with wild-type (wt) and mutated (mut) ALAS2 IRE. Graphic representation of the ferritin $\mathrm{H}$ (FTH1) IRE sequences (nucleotides 60-85 in NM_002032.2) wt or mut -165 $\mathrm{C}$, ALAS2 IRE sequence (nucleotides 93-125 in NM_000032.4) wt or the -38U>C mut, used for the synthesis of RNA probes in competitive EMSA experiment. RNA Watson-Crick pairs are depicted as A-U; U-A; C-G or G-C; wobble pairings are shown as U.G and not possible pairings are depicted as CXA. Fluorescent-labeled FTH1 IRE wt probe was incubated with increasing molar excess (2x, 5x, 10x, 20x, 40x and 80x) of unlabeled competitors corresponding to the FTH1 IRE wt type sequence (lanes 3-8; lanes 31-26), or the FTH1 IRE mutant -165 $\Delta C$ (lanes 9-14; lanes 37-42), ALAS2 IRE wt sequence (lanes 17-22; lanes 37-42) or the ALAS2 -38U>C mutation (lanes 23-28; lanes 51-56). Samples were incubated with recombinant IRP1 (upper panel) or IRP2 (bottom panel) and resolved on acrylamide gels. Quantification of the signals of the shifted bands was performed using the Odyssey Infrared Imaging System (LI-COR Biosciences) and compared to the signal in lane N, set as $100 \%$. Means \pm standard deviation of at least three independent experiments. Statistical analysis by Student's $t$-test (two-tailed) compares the signal given by the mutated IRE of FTH1 or ALAS2 sequences to the signal given by the corresponding wt IRE sequences, at each molar concentration $* P<0.05, * * P<0.01$, $* * \star P<0.001$. F: free probe; N: no competitor added. (C) Proteins from CD34 $4^{+}$erythroid cells from proband's mother (II.5) and the proband (III.2) cultured and differentiated over 14 days were extracted. Twenty micrograms were separated (4-12\% NuPAGE gel) with MES NuPAGE buffer. Proteins were transferred to nitrocellulose membrane. ALAS2 was detected by chemiluminescence using specific antibody. The lower band is ALAS2 whereas the upper band, indicated with an asterix, is an unknown non-specific protein (Agios Pharmaceutical). The membrane was then probed with an anti-human beta-actin antibody as a loading control.

nucleotide substitution (c.1102G>A; p.G298D) at the exon 7 of CLPX gene. ${ }^{11}$

The proband did not harbor mutations in the $11^{\text {th }}$ exon of $A L A S 2$ gene demonstrating that she is affected by an unusual form of EPP. Sequencing of the rest of the ALAS2 gene revealed an heterozygous $\mathrm{T}>\mathrm{C}$ change at position -38 in the first exon, at the 5 ' UTR and located inside the ALAS2 IRE motif (NM_000032.4; c.[$38 \mathrm{~T}>\mathrm{C}] ;[=]$ ) (Figure $1 \mathrm{~A}$ and $\mathrm{B}$ ). The mutation is absent in the 6,500 sequenced exomes in the Exome Variant Server database, excluding the possibility of being a neutral polymorphism. This variation was predicted to disturb the stability of the IRE, since the IRE position below the C 8 bulge is critical to maintain closed the lower stem (IRE ALAS2 wild-tytpe [wt] predicted minimum free energy $\Delta \mathrm{G}=-7.00 \mathrm{Kcal} / \mathrm{mol}$ vs. IRE $A L A S 2$ mutant predicted minimum free energy $\Delta \mathrm{G}=-5.60$
$\mathrm{Kcal} / \mathrm{mol}){ }^{12}$ In order to confirm the functional consequence of the $-38 \mathrm{U}>\mathrm{C}$ change in ALAS2 IRE, we performed non-radioactive competitive electrophoretic mobility shift assays (EMSA) with recombinant IRP1 or IRP $2^{13}$ (Figure 1B). As expected, the ferritin H (FTH1) wt positive control showed efficient competition, while its corresponding negative control, the FTH1 mutant $165 \Delta \mathrm{C}$ could not compete with the labeled FTH1 wt probe (Figure 1B, compare lanes 3-8 to $9-14$ in upper panel and 31-36 to 37-42 in bottom panel). In addition, the ALAS2 -38U>C mutation totally abolished the ability of the ALAS2 IRE to compete with FTH1 wt probe for IRP1 or IRP2 binding, in contrast to the wt ALAS2 IRE sequence (Figure 1B, compare lanes $17-22$ to $23-28$ in upper panel and $45-50$ to $51-56$ in bottom panel). Therefore, the $-38 \mathrm{U}>\mathrm{C}$ mutation in ALAS2 IRE abolishes IRP1 and IRP2 binding affinity in vitro, which would 
impair ALAS2 mRNA regulation in response to iron levels. We hypothesized that under iron limiting conditions, IRP would not repress ALAS2 mRNA translation, with consequent overproduction of ALAS2 protein. This predicted increase in ALAS2 protein levels was indeed observed by immunoblotting for ALAS2 from cell lysates obtained from cultured and differentiated $\mathrm{CD}_{3} 4^{+}$erythroid precursors from the proband and the mother (II.5) who only bears the ALAS2 IRE mutation (Figure 1C). Therefore, our data supports the hypothesis that the $38 \mathrm{U}>\mathrm{C}$ mutation in the ALAS2 IRE increases ALAS2 protein expression and modifies the severity of EPP caused by a confirmed CLPX mutation. In contrast, the ALAS2 gain-of-function in XLPP is caused by increased enzymatic activity in the protein. ${ }^{14}$

Sequencing of the ALAS2 gene in other family members showed that this mutation does not co-segregate with porphyrin accumulation (Figure $1 \mathrm{~A}$ and data not shown). The proband inherited the $-38 \mathrm{U}>\mathrm{C}$ change from the maternal branch (II:5; II:6; I3, Figure 1A). Those relatives have higher than normal $\mathrm{ZnPP}$ levels and are asymptomatic (Table 1). Methylation analysis confirmed the absence of skewed $\mathrm{X}$ inactivation in relatives (II.5 and II.6) and the proband (III.2) who carried the IRE mutation.

Previously we and others described the important role of CLPX in heme biosynthesis through its regulation of ALAS2 turnover and enzyme activity. ${ }^{11,15}$ The G298D mutation in the CLPX gene, present in heterozygosity in this family (proband, father and paternal uncle), decreases the proteolytic activity of the CLPXP protease, causing accumulation of ALAS2 ${ }^{11}$ However, in comparison to the mild photosensitivity presented by the paternal family, the proband presents with a full and severe EPP phenotype due to the combination of the paternal CLPX G298D mutation and the maternal ALAS2 -38T>C mutation present in the 5' IRE of ALAS2 gene.

Collectively, our data suggests that the ALAS2 IRE mutation is the modifier necessary for the clinical presentation of EPP in the proband. The IRE/IRP-dependent down regulation of $A L A S 2$ during iron deficiency is impaired and contributes to ALA overproduction and increases PPIX level in erythrocytes especially during iron deficiency. This postulate was supported clinically by the beneficial effect of the oral iron therapy when the patient was anemic (Table 1).

In summary, we have identified the first described mutation in the IRE of ALAS2 gene that contributes to porphyrin accumulation in erythrocytes above a threshold leading to overt EPP. These data strongly support the role of $A L A S 2$ as an important modifier gene triggering the clinical manifestations in erythropoietic disorders and extend the involvement of its IRE/IRP system to human heme metabolism and erythropoietic porphyria.

Sarah Ducamp ${ }^{1,2^{*}}$ Sara Luscieti, ${ }^{3^{*}}$ Xènia Ferrer-Cortès,${ }^{4,5}$ Gaël Nicolas, ${ }^{1,2}$ Hana Manceau, ${ }^{2,6}$ Katell Peoc'h, ${ }^{2,6}$ Yvette Y. Yien, ${ }^{7}$ Caroline Kannengiesser, ${ }^{1,3}$ Laurent Gouya, ${ }^{1,2,6}$ Herve Puy, ${ }^{1,2,6 \#}$ and Mayka Sanchez $z^{4,5 \#}$

${ }^{1}$ Centre de Recherche sur l'inflammation, INSERM U1149 CNRS ERL Université Paris Diderot, site Bichat, Sorbonne Paris Cité, Paris, France; ${ }^{2}$ Laboratory of Excellence, GR-EX, Paris, France; ${ }^{3}$ Institute of Predictive and Personalized Medicine of Cancer (IMPPC), Badalona, Barcelona, Spain; ${ }^{4}$ Universitat Internacional de Catalunya (UIC), Department of Basic Sciences, Iron metabolism: Regulation and Diseases, Sant Cugat del Vallès, Barcelona, Spain; ${ }^{5}$ BloodGenetics S.L. Diagnostics in Inherited
Blood Diseases, Espluques de Llobregat, Barcelona; Spain;

${ }^{6}$ Centre Français des Porphyries, AP-HP, Hôpital Louis Mourier,

Colombes, France and ${ }^{7}$ Department of Biological Sciences,

University of Delaware, Newark, DE, USA

* $S D$ and SL contributed equally as co-first authors.

"HP and MS contributed eugally as co-senior authors.

Correspondence: HERVE'PUY - herve.puy@aphp.fr

MAYKA SANCHEZ - msanchez@uic.es

doi:10.3324/haematol.2020.272450

Received: September 23, 2020.

Accepted: January 22, 2021.

Pre-published: Fabruary 18, 2021

Disclosures: no conflicts of interest to disclose.

Contributions: MS, LG and HP developed the study concept and designed the research; KP, LG and HP recruited family members and collected patients' data; SD and HM performed patients DNA sequencing; Ckdid the X inactivation and CGH array experiments;

SL performed the IRE/IRP functional EMSA; HM did the erythroid progenitor cell culture; GN perfomed immunoblot experiments;

$X F-C, Y Y Y, H P$ and MS wrote the manuscript and all authors participated in data discussion, analyzed data, read and approved the manuscript.

Acknowledgments: we are very grateful to all parent family who kindly contributed to this study. We thank Drs. Carole Beaumont, Zoubida Karim and Säid Lyoumi for helpful feedback, discussions, and editorial assistance.

Funding: this study was partially supported by grant SAF2015-70412-R, and grant RTI-2018-101735-B-I100, MCI/AEI/FEDER, EU from the Spanish Secretary of Research, Development and Innovation (MINECO); grant DJCLS-R-14/04 from the Deutsche Josep Carreras Leukämie-Stiftung, 2014 SGR225 (GRE) from Generalitat de Catalunya to MS; SD and $H M$ were supported by the Laboratory of excellence, $B R-E x$, Paris, France;

the labex GR-Ex (reference ANR-11-LABX-0061) is funded by the program "Investissements d'avenir" of the French National Research Agency (reference ANR-11-IDEX-0005-02); YYY is supported by the National Institutes of Health (R03DK118307).

\section{References}

1.Puy H, Gouya L, Deybach JC. Porphyrias. Lancet. 2010;375(9718):924-937.

2. Balwani M, Desnick RJ. The porphyrias: advances in diagnosis and treatment. Blood. 2012;120(23):4496-4504.

3. Gouya L, Martin-Schmitt C, Robreau AM, et al. Contribution of a common single-nucleotide polymorphism to the genetic predisposition for erythropoietic protoporphyria. Am J Hum Genet. 2006;78(1):2-14

4. Whatley SD, Ducamp S, Gouya L, et al. C-terminal deletions in the ALAS2 gene lead to gain of function and cause X-linked dominant protoporphyria without anemia or iron overload. Am J Hum Genet. 2008;83(3):408-414.

5. Ducamp S, Schneider-Yin X, de Rooij F, et al. Molecular and functional analysis of the C-terminal region of human erythroid-specific 5-aminolevulinic synthase associated with X-linked dominant protoporphyria (XLDPP). Hum Mol Genet. 2013;22(7):12801288

6. To-Figueras J, Ducamp S, Clayton J, et al. ALAS2 acts as a modifier gene in patients with congenital erythropoietic porphyria. Blood. 2011;118(6):1443-1451.

7. Dandekar T, Stripecke R, Gray NK, et al. Identification of a novel iron-responsive element in murine and human erythroid deltaaminolevulinic acid synthase mRNA. EMBO J. 1991;10(7):19031909

8. Beaumont C, Leneuve P, Devaux I, et al. Mutation in the iron responsive element of the $\mathrm{L}$ ferritin $\mathrm{mRNA}$ in a family with dom- 
inant hyperferritinaemia and cataract. Nat Genet. 1995;11(4): 444-446.

9. Girelli D, Corrocher R, Bisceglia L, et al. Molecular basis for the recently described hereditary hyperferritinemia-cataract syndrome: a mutation in the iron-responsive element of ferritin Lsubunit gene (the "Verona mutation"). Blood. 1995;86(11):40504053.

10. Kato J, Fujikawa K, Kanda M, et al. A mutation, in the ironresponsive element of $\mathrm{H}$ ferritin mRNA, causing autosomal dominant iron overload. Am J Hum Genet. 2001;69(1):191-197.

11. Yien YY, Ducamp S, van der Vorm LN, et al. Mutation in human CLPX elevates levels of $\delta$-aminolevulinate synthase and protoporphyrin IX to promote erythropoietic protoporphyria. Proc Natl Acad Sci U S A. 2017;114(38): e8045-e8052.
12. Campillos M, Cases I, Hentze MW, Sanchez M. SIREs: searching for iron-responsive elements. Nucleic Acids Res. 2010;38 (Suppl):W360-367.

13. Luscieti S, Tolle G, Aranda J, et al. Novel mutations in the ferritin-L iron-responsive element that only mildly impair IRP binding cause hereditary hyperferritinaemia cataract syndrome. Orphanet J Rare Dis. 2013;8(1):30.

14. Fratz EJ, Clayton J, Hunter GA, et al. Human erythroid 5aminolevulinate synthase mutations associated with X-linked protoporphyria disrupt the conformational equilibrium and enhance product release. Biochemistry. 2015;54(36):5617-5631.

15. Whitman JC, Paw BH, Chung J. The role of ClpX in erythropoietic protoporphyria. Hematol Transfus Cell Ther. 2018;40(2):182188. 